\title{
On the use of the modulated reflectance microscopy in the study of laser diode facets: detection of surface defects
}

\author{
A.M. Mansanares(1), J.P. Roger, D. Fournier and A.C. Boccara \\ Laboratoire d'Optique Physique, ESPCI, UPR A0005 du CNRS, Laboratoire d'Instrumentation, UPMC, \\ UPR A0005 du CNRS, 10 rue Vauquelin, 75005 Paris, France
}

\begin{abstract}
InGaAsP/InP buried heterostructure lasers were investigated using reflectance microscopy. Measurements were performed for both biased (current modulation) and non-biased (modulated pump beam) device. Maps of the facets of non-degraded and degraded lasers are presented and discussed.
\end{abstract}

\section{INTRODUCTION AND EXPERIMENTAL ARRANGEMENT}

In modulated reflectance microscopy a continuous laser beam $(670 \mathrm{~nm})$ is used to probe the sample reflectance variation induced by thermal and plasma waves in the sample, which are created by an external excitation [1-2]. The probe beam is focused on the sample surface by a microscope (50x objective, N.A. $=0.75$ ) with a diameter of about $1.0 \mu \mathrm{m}$ and its incident power is of a few hundreds of $\mu \mathrm{W}$. The beam is reflected back by the surface and deviated in the direction of a $\mathrm{Si}$ photodiode by using a $\lambda / 4$ plate combined with a polarizing beam splitter cube. The sample is mounted on an $\mathrm{x}-\mathrm{y}$ translation stage (step-size $0.1 \mu \mathrm{m}$ ) allowing the probe beam to scan its surface. In conventional experiments the excitation is provided by an intensity modulated pump beam (514.5 $\mathrm{nm} \mathrm{Ar}^{+}$laser of $1.0 \mu \mathrm{m}$ diameter) focused on the sample surface at the same position of the probe beam. In this case, the maps obtained when scanning the sample are related to the spatial distribution of its physical properties, such as thermal conductivity and diffusivity, optical absorption coefficient, energy band-gap, etc. [3] On the other hand, when investigating electronic and optoelectronic devices, the excitation can be given by the injected current for device operation itself. In this configuration, the measurement maps reveal the actual distribution of temperature and carrier density of the operating device $[1,2,4,5]$. This strategy allows us to detect critical points, such as overheating points, displaying local defects. In both cases, high modulation frequencies (f, $\mathrm{MHz}$ range) are used in order to impose a small thermal diffusion length (of a few microns), thus enhancing the signal phase contrast.

The output signal of the $\mathrm{Si}$ photodiode is proportional to the reflected probe beam power, which depends on the sample reflectance (R). When modulating the pump beam or the injection current, a small perturbation $\Delta \mathrm{R}$ is added to the sample reflectance $R$, the perturbation being modulated at the same frequency. Then, measuring the ac component of the $\mathrm{Si}$ photodiode output signal, using a lock-in amplifier, and normalizing it by the dc component, we found the experimental ratio $(\Delta R / R)$, which depends on the sample temperature variation $(\Delta T)$ and carrier density variation $(\Delta N)$ in the following way:

$$
\frac{\Delta \mathrm{R}}{\mathrm{R}}=\left(\frac{1}{\mathrm{R}} \frac{\partial \mathrm{R}}{\partial \mathrm{T}}\right) \Delta \mathrm{T}+\left(\frac{1}{\mathrm{R}} \frac{\partial \mathrm{R}}{\partial \mathrm{N}}\right) \Delta \mathrm{N}
$$

The coefficients $(1 / R)(\not R / \partial X), X=T, N$ are characteristic of the material and depend on the probe wavelength. 
In this work we have investigated cleaved, uncoated, InGaAsP/InP distributed feedback buried heterostructure (DFB-BH) lasers. The lasers are $215 \mu \mathrm{m}$ long and operate at $1.55 \mu \mathrm{m}$. The active layer $\left(\lambda_{\text {gap }}=1.55 \mu \mathrm{m}\right)$ is $100-220 \mathrm{~nm}$ thick and $(2 \pm 0.5) \mu \mathrm{m}$ wide. Maps of the laser facet were obtained for both non-biased (pump beam modulation) and biased device (current modulation).

\section{RESULTS AND DISCUSSION}

As mentioned above, the dc component of the $\mathrm{Si}$ photodiode output signal is proportional to the sample reflectance $\mathrm{R}$ at the probe wavelength. Figure 1 shows a map of the dc signal $\left(D_{670 \mathrm{~nm}}\right)$ around the laser facet. We can observe the different levels of reflectance of the InP substrate, the active region and the re-grown InP layers. The peak of reflectance in the active region is due to the lower energy band-gap of the quaternary InGaAsP. We must note that this map represents the convolution of the local sample reflectance by the probe beam intensity distribution.



Fig. 1: dc component of the reflectance signal around the laser facet. The same orientation is used in the 2-D scans shown in the next figures.

In Fig. 2 we show the reflectance image when scanning the laser facet with the $\mathrm{Ar}^{+}$laser used as pump beam. Incident pump power was of a few $\mathrm{mW}$ and modulation frequency of $1.0 \mathrm{MHz}$. We observe a high signal amplitude in the active region, and an almost constant amplitude level in the InP regions, with a small contrast between the substrate and the re-grown InP layers. Signal phase map (see line scan in Fig. 3) reveals a near zero phase value in the entire scanned area. It means that the temperature contribution to the reflectance signal is dominant at this modulation frequency for all regions, since $(\partial R / \partial T)$ is positive, giving a signal phase around zero, while $(\not R / \partial \mathbb{N})$ is negative and would give a signal phase near $180^{\circ}[3,6]$. The peak in the active layer is due to the lower energy band-gap and thermal conductivity of the quaternary. The band-gap of the active region is $0.799 \mathrm{eV}$ and the InP one is $1.35 \mathrm{eV} \mathrm{[7].} \mathrm{So,} \mathrm{the}$ released energy in the relaxation to the bottom of the band, after photon absorption, is greater in the active layer than in the InP regions, resulting in a higher temperature variation. Moreover, the temperature $\Delta \mathrm{T}$ is inversely proportional to the thermal conductivity, enhancing the temperature contrast between InP and active region $(\mathrm{k}=680 \mathrm{~mW} / \mathrm{cmK}$ for InP, and $\mathrm{k}=50.6 \mathrm{~mW} / \mathrm{cmK}$ for the active layer [7]).

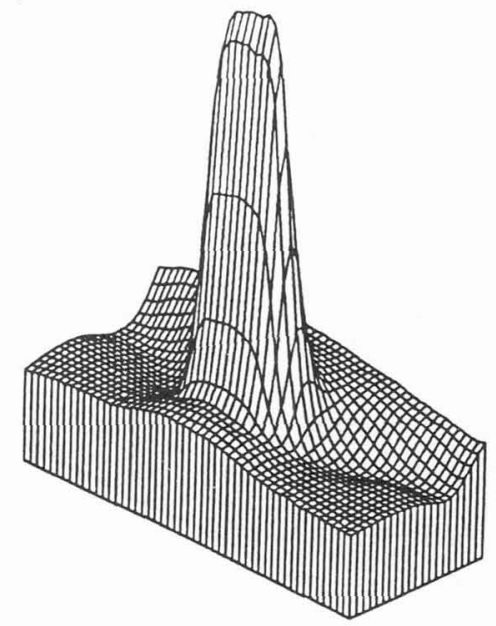

Fig. 2: Amplitude of the photothermal reflectance signal on the laser facet. The modulation frequency of the $\mathrm{Ar}^{+}$laser beam was $1.0 \mathrm{MHz}$. The baseline is at zero amplitude, and the maximum $\triangle R / R$ is $2.5 \times 10^{-3}$. Scanned area is $5.0 \mu \mathrm{m} \times 2.0 \mu \mathrm{m}$.

When increasing the modulation frequency to 10.0 $\mathrm{MHz}$, the excitation period becomes of the order of the photo injected carriers lifetime, and in this case, the carrier contribution to the signal will be relevant. In Fig. 3 we show the signal phase when scanning on the laser facet, parallel to the active layer, for both $1.0 \mathrm{MHz}$ and $10.0 \mathrm{MHz}$. We can see that the carrier contribution is enhanced in the quaternary region at $10.0 \mathrm{MHz}$ (phase dropping to 290 degrees). This is due to the carrier confinement produced by the band-gap distribution (heterojunction), which imposes a quasi-onedimensional carrier diffusion. The resulting carrier density is then increased in the quaternary material.

Let us now discuss the results obtained for the biased device. Here an injection current of the form $I(t)=I_{d c}+I_{a c} \sin (2 \pi f t)$ was used. A typical map 
is shown in Fig. 4 for the signal amplitude. The modulation frequency was $1.0 \mathrm{MHz}, \mathrm{I}_{\mathrm{dc}}$ and $\mathrm{I}_{\mathrm{ac}}$ were $70 \mathrm{~mA}$ and $20 \mathrm{~mA}$, respectively (the threshold current of the laser is $42 \mathrm{~mA}$, thus the device is always operating). From the phase analysis we can conclude that the reflectance variation is mainly due to temperature changes (see discussion above for the $\mathrm{Ar}^{+}$laser excitation case). The amplitude map shows a hot spot in the active zone. The strong thermal mismatch between InGaAsP and InP induces a heat confinement in the active region, originating the sharp temperature distribution observed in Fig. 4. Furthermore, when device operates, the laser mode guides the thermal losses to the center of the active layer. A wider heat source distribution is observed when device is biased below threshold. In that case, the obtained map resembles those of Figs. 1 and 2. A detailed discussion of the shape of the temperature distribution of the operating laser is presented in Refs. 2 and 3.

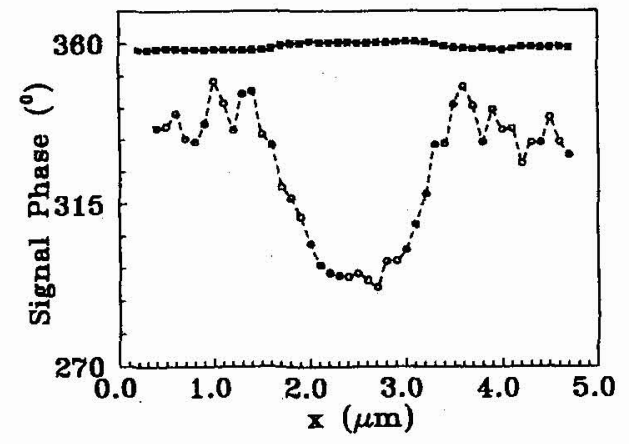

Fig. 3: Signal phase on the laser facet, parallel to the active layer, at $1.0 \mathrm{MHz}$ (squares) and 10.0 $\mathrm{MHz}$ (circles). Ar laser was used as pump beam.

Up to now we presented results obtained on the facet of lasers that did not undergo any kind of degradation. In the following discussion we would like to consider the case of laser facet damage. Catastrophic optical mirror damage (COMD) in laser diodes seems to be the result of strong heating of the crystal facet due to laser operation, with temperature reaching the materials melting point. In this case structural transformation may take place, resulting in dark regions, i.e., regions that do not participate in laser oscillation, due to changes in their energy band-gap [8].

The results we are going to present here were obtained on the same laser of Figs. 1 to 4 , after undergoing $\mathrm{COMD}$ induced by an $\mathrm{Ar}^{+}$laser beam incidence on its facet during device operation. A point defect was created, as we can see in Figs. 5 to 7 , and the laser threshold current has shifted from $42 \mathrm{~mA}$ to $47 \mathrm{~mA}$, indicating that a fraction of the injected carriers is being lost in the defect (it does not contribute to the laser emission).

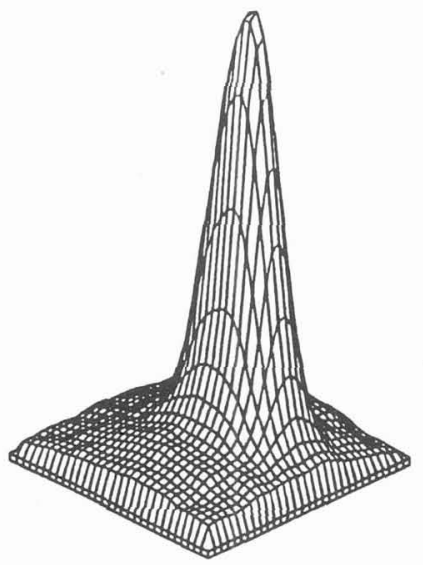

Fig. 4: Amplitude of the photothermal reflectance signal on the laser facet. The modulation frequency was $1.0 \mathrm{MHz}$. The device was biased with a dc current of $70 \mathrm{~mA}$ and an ac current of $20 \mathrm{~mA}$. The baseline is at zero amplitude, and the maximum $\triangle R R$ is $7.6 \times 10^{-4}$. Scanned area is $3.0 \mu \mathrm{m} \times 3.0 \mu \mathrm{m}$.

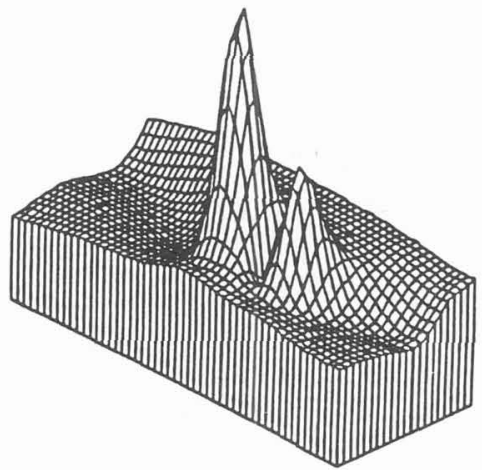

Fig. 5: Amplitude of the photothermal reflectance signal on the laser facet, after COMD. The modulation frequency of the $\mathrm{Ar}^{+}$laser beam was 1.0 $\mathrm{MHz}$. The baseline is at zero amplitude, and the maximum $A R R$ is $1.7 \times 10^{-3}$. Scanned area is $5.0 \mu \mathrm{m} \times 2.0 \mu \mathrm{m}$.

Figure 5 shows the signal amplitude when scanning the laser facet with the $\mathrm{Ar}^{+}$laser used as pump beam (the same experimental conditions of Fig. 2 were employed; $\mathrm{f}=1.0 \mathrm{MHz}$ ). We can 
observe that at the position of the defect the signal amplitude drops to the InP signal level. Phase does not change significantly in the entire scanned area, as in the case of Fig. 3. It is conceivable that a structural transformation could move the energy band-gap and the thermal properties in the InP direction. This hypothesis is supported by further results, as discussed below.

In Fig. 6 we show the amplitude map for the biased device, in the same experimental conditions described in Fig 4 ( $f=1.0 \mathrm{MHz}, I_{D C}=70 \mathrm{~mA}$, $I_{A C}=20 \mathrm{~mA}$ ). In this case we see that the signal amplitude also falls in the defect region (see the line scan parallel to the active layer in Fig. 7). Analyzing the phase behavior, we verify that at the defect region it shifts about $120^{\circ}$ (Fig. 7), indicating that the carrier contribution to the reflectance signal becomes important at the defect. Indeed, the decrease in signal amplitude should be due to the fact that one of the contributions to the signal (temperature or carrier density) is being almost canceled out by the other one. It means that the injected current is creating a local carrier density at the defect higher than in the normal (nondegraded) active region. It can be explained if we consider a longer carrier lifetime in the defect than in the active layer. This hypothesis is partially satisfied if the defect region actually does not participate in the stimulated emission.

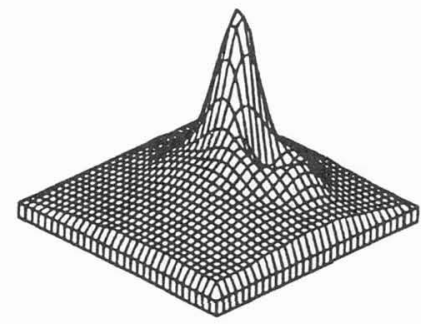

Fig. 6: Amplitude of the photothermal reflectance signal on the laser facet after COMD. The modulation frequency was $1.0 \mathrm{MHz}$. The device was biased with a dc current of $70 \mathrm{~mA}$ and an ac current of $20 \mathrm{~mA}$. The baseline is at zero amplitude, and the maximum $\triangle R R$ is $2.8 \times 10^{-4}$. Scanned area is $3.0 \mu \mathrm{m} \times 3.0 \mu \mathrm{m}$.

In conclusion, we have used reflectance microscopy in the investigation of laser diode mirrors. Two different excitation sources were used (modulated pump beam and current modulation). Comparison between non-degraded and degraded surfaces provided information on the nature of the local defect.

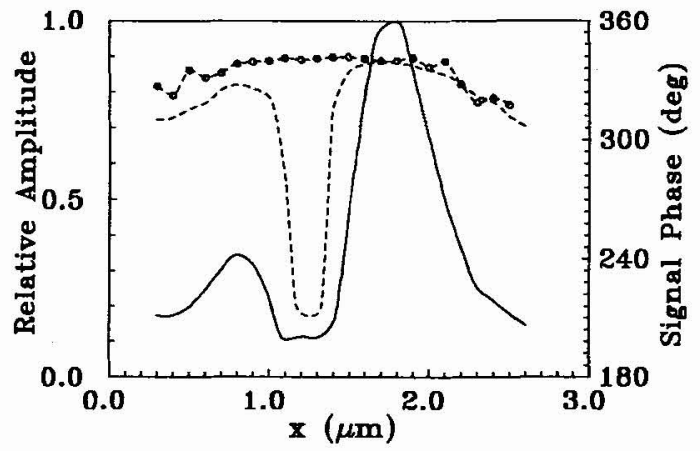

Fig. 7: Amplitude (solid line) and phase (dashed line) of the photothermal reflectance signal, after COMD, when scanning the laser facet in the direction parallel to the active layer (experimental conditions described in Fig. 6). Circles represent the signal phase before laser degradation.

A.M.M. is grateful to the Brazilian Agencies $\mathrm{CNPq}$ and FAPESP.

\section{REFERENCES}

[1] Mansanares A.M., Roger J.P., Fournier D. and Boccara A.C., Appl. Phys. Lett. (1994).

[2] Mansanares A.M., Fournier D. and Boccara A.C., Electronics Lett. 29 (1993) 2045-2047.

[3] Inglehart L.J., Broniatowski A., Fournier D., Boccara A.C. and Lepoutre F., Appl. Phys. Lett. 56 (1990) 1749-1751.

[4] Epperlein P. W., Bona G. L. and Roentgen P., Appl. Phys. Lett. 60 (1992) 680-682.

[5] Epperlein P. W. and Bona G. L., Appl. Phys. Lett. 62 (1993) 3074-3076.

[6] Rosencwaig A., "Thermal Wave Characterization and Inspection of Semiconductor Materials and Devices", Photoacoustic and Thermal Wave Phenomena in Semiconductors (North-Holland, New York, 1987) pp. 97-135.

[7] Nakwaski W., J. Appl. Phys. 64 (1988) 159166.

[8] Todoroki, S., J. Appl. Phys. 60 (1986) 61-65. 